\title{
Detection of cancer specific mutations in early-stage non-small cell lung cancer using cell-free DNA by targeted sequencing
}

\author{
YUTONG HE $^{1}$, XINYUAN ZHANG ${ }^{2 *}$, LIQUN WANG ${ }^{1}$, ZIQIANG TIAN $^{1}$, QINGYI LIU $^{1}$, \\ JIFANG YAO ${ }^{1}$, YUEPING LIU ${ }^{1}$, CHUANBAO LI ${ }^{3}$, LI MIN $^{4}$ and BAOEN SHAN ${ }^{1}$ \\ ${ }^{1}$ The Fourth Hospital of Hebei Medical University/The Tumor Hospital of Hebei Province, \\ Shijiazhuang, Hebei 050011; ${ }^{2}$ Institute of Genecrab Precision Medicine, \\ Beijing 100078; ${ }^{3}$ Department of Clinical Laboratory, Beijing Hospital, \\ Beijing 100730; ${ }^{4}$ Department of Biochemistry and Molecular Biology, \\ Peking University Cancer Hospital and Institute, Beijing 100142, P.R. China
}

Received August 2, 2016; Accepted September 5, 2016

DOI: $10.3892 /$ ijo.2016.3731

\begin{abstract}
Non-small cell lung cancer (NSCLC) is a major public health problem worldwide and leads to a high mortality. NSCLC is always diagnosed in late stages because of its unapparent symptoms. However, cell-free DNA (cfDNA) may serve as a new potential biomarker to detect early stage of non-small cell lung cancer. Here we recruited 10 non-small cell lung cancer patients to obtain fresh tumor tissue, peripheral blood lymphocytes (PBLs), and plasma. CfDNAs from 13 elderly people and 7 middle-age smokers were also extracted as controls. Illumina HiSeq X10 was used to perform nextgeneration sequencing to evaluate differences in mutations among different samples. The result indicated that tumor DNA and its matched plasma cfDNA samples showed high concordance in their mutation patterns. Mutation rate of cfDNA was generally lower than that of tumor tissue and higher than that of PBLs. The plasma cfDNA concentration of NSCLC patients $(69.2 \pm 46.9 \mathrm{ng} / \mathrm{ml})$ is significantly higher than that of elderly people $(32.5 \pm 5.2 \mathrm{ng} / \mathrm{ml}, \mathrm{t}=2.96, \mathrm{p}=0.007)$ and middle-aged smokers $(17.9 \pm 9.1 \mathrm{ng} / \mathrm{ml}, \mathrm{t}=2.83, \mathrm{p}=0.013)$. Five mutations (PTEN_c.1375A > G, TP53_c.94G >A, STK11_c.816C >T, PIK3CA_c.1633 G>A, PIK3CA_c.2038G>C) were only identified in NSCLC patients but not in healthy people. Our conclusion was that cfDNA has a similar mutation pattern with its matched tumor tissue DNA. A high concentration of cfDNA and tumor specific mutations in cfDNA may serve
\end{abstract}

Correspondence to: Dr Baoen Shan, The Fourth Hospital of Hebei Medical University/The Tumor Hospital of Hebei Province, Shijiazhuang, Hebei 050011, P.R. China

E-mail: baoenshan@hbydsy.com

*Contributed equally

Key words: cell-free DNA, non-small cell lung cancer, mutations, targeted sequencing as potential non-invasive biomarkers to detect early-stage non-small cell lung cancer.

\section{Introduction}

It is reported that there were 1.8 million new cases of lung cancer in 2012 (12.9\% of the total), 58\% of which occurred in less developed regions (1). Lung cancer is estimated to be responsible for nearly one in five cancer related deaths worldwide (1.59 million deaths, $19.4 \%$ of the total), which makes it a major public health problem (2).

In most Western countries, lung cancer incidence and death rates are decreasing in men and plateauing in women $(3,4)$; however, both incidence and mortality rates of lung cancer are still increasing in China and there were $\sim 652,800$ new cases and 597,200 deaths in China in 2015, accounting for $35.78 \%$ and $37.56 \%$ of the whole world (5). Approximately $85 \%$ of patients diagnosed with lung cancer are non-small cell lung cancer (NSCLC) and two in three of these cases are diagnosed in metastatic or advanced stages (6). NSCLC patients with early stage could have better opportunity of admirable survival (7). Therefore minimal damage, effective and convenient detection for early stage of non-small cell lung cancer is particularly necessary.

Currently there are several methods to detect NSCLC, such as tissue biopsy, which is the golden standard of diagnosis. However, it is often difficult to obtain biopsy samples, and it is very challenging or time-consuming to acquire samples especially in early-stage cases from different medical centers. Low-dose computed tomography (LDCT) and X-ray examination are two other main approaches. LDCT is an ever emerged National Lung Screening Trial (NLST) in US; however, LDCT screening has a relatively low specificity $(73.4 \%)$, which results a high false-positive findings rate (96.4\%) (8). LDCT screening is simple and was shown to confer a $20 \%$ reduction in lung cancer mortality and a higher proportion of early NSCLC diagnosis in a highrisk population (9), but its utility and validity are still under debate (10-12). Recently the U.S. Preventive Services Task 
Force recommendation statement (USPSTF) recommended annual LDCT-screening for lung cancer in high-risk individuals and stressed the need for more research into the use of biomarkers to complement LDCT screening.

CfDNA corresponds to cell-free DNA fragments circulating in the bloodstream which can be extracted from plasma or serum. CfDNA is mostly composed of constitutive genomic DNA (13). One of the most immediate applications of circulating cfDNA has been termed 'liquid biopsy' in research studies as well as in clinical practices $(14,15)$. Detection of genetic and specific mutations will be the most promising tool for large-scale population-based lung cancer screening when considering its safety, availability and accuracy (11). Sun et al found that compared with other non-invasive approaches to monitor EGFR-TKI treatment in NSCLC patients, cfDNA displayed many advantages: moderate sensitivity, high specificity, feasible on small-amount samples, rapid and low cost, and high reproducibility (16). Identified mutations in cfDNA have also been found to be potential prognostic biomarkers of NSCLC $(17,18)$. Here we expanded the increasing interest in this approach to explore potential application in early stage screening and diagnosis of NSCLC patients.

\section{Materials and methods}

Patient information and ethics statement. Tumor and blood samples from 10 NSCLC patients were analyzed in this study. All patients, including 8 males and 2 females with an average age of $57.8 \pm 8.88$, were diagnosed with stage IA, IB, IIA, and IIB NSCLC, of these 5 were adenocarcinoma, 4 were squamous cell carcinoma (SCC), and 1 was sarcomatoid carcinoma. Three of these patients have direct relatives diagnosed with cancer. Four of these patients have long term smoking history (>20 years). 20 healthy controls, including 13 elderly people and 7 middle-age smokers with an average age of $66.8 \pm 15.46$, were also recruited from noncancerous out-patients of Hebei Medical University Fourth Hospital. The study was approved by Ethics Committees of Hebei Medical University Fourth Hospital. All participants, including NSCLC patients and healthy controls, provided written informed consent for this study.

Sample DNA handling. Fresh tumor tissue, peripheral blood lymphocytes (PBLs), and plasma were collected for analysis for each patient. Tubes $(10 \mathrm{ml})$ containing blood samples with EDTA added were centrifuged at $1000 \mathrm{~g}$ for $10 \mathrm{~min}$. The cell pellets containing peripheral blood lymphocytes were stored at $-20^{\circ} \mathrm{C}$. The supernatants were centrifuged again at $10,000 \mathrm{~g}$ for $10 \mathrm{~min}$, and plasma was collected and stored at $-80^{\circ} \mathrm{C}$. Tiangen tissue DNA kit (Tiangen, Beijing, China) and Tiangen whole blood DNA kit (Tiangen) were used to extracted DNA from fresh tumor tissue and peripheral blood lymphocytes, respectively. QIAamp Circulating Nucleic Acid kit (Qiagen, Germany) was used to extract cfDNA form plasma. All kits were used according to the manufacturer's instructions.

Library preparation and sequencing. For each sample, DNA was quantified with the Qubit dsDNA HS Assay kit (Life Technologies, USA) according to the manufacturer's instructions. Targeted amplification and Illumina adapterligated library preparation was performed using Amplicon Sequencing-Illumina Compatible kit following the manufacturer's instructions (Genecrab, Beijng, China). All samples were subjected to Illumina HiSeq X-Ten for paired-end sequencing (150 bp each end). The AmpliSeq Cancer Panel covers 92 continuous region with 10,235 bp in 22 cancerassociated genes (KRAS, EGFR, BRAF, PIK3CA, AKT1, ERBB2, PTEN, NRAS, STK11, MAP2K1, ALK, DDR2, CTNNB1, MET, TP53, SMAD4, FBX7, FGFR3, NOTCH1, ERBB4, FGFR1 and FGFR2), which developed by Life Technologies Co.

Variant calling. Initial data from HiSeq X-Ten were evaluated by using fastQC (v0.11.3). Raw reads were mapped to reference genome hg19 by using BWA (0.7.12-r1039). Program Samtools and VarScan (v2.4.1) was used for variant calling: i) the average total coverage depth was defined as $>1,000$ and each variant coverage as $>10$; for called variant, at least one sample with variant frequency $>1 \%$, variant frequency of each sample $>0.5 \%$, and P-value $<0.01$; ii) visual examination of the mutations was performed using Samtools software (http://samtools. sourceforge.net) and possible errors specific to one DNA strand were filtered out. Software ANNOVAR (v2015-06-17) and snpEff (v4.2) was used for variant annotation.

Statistical analysis. For variant frequency $<0.5 \%, 0$ was replaced. R (hclust, v3.2.4) was used for variant frequency clustering analysis to show the types of samples from cancer patients that are more similar. Student's t-test was applied for comparison of cfDNA concentration and $\mathrm{p}<0.05$ was considered statistically significant.

\section{Results}

Sequence $Q C$ with the Illumina X10. For the 10 NSCLC patients, tumor DNA, matched blood DNA, and plasma cfDNA were all subjected to sequencing. Of all 30 sequenced samples, sequence lengths were all set to $150 \mathrm{bp}$. The quality scores were $\sim 40$, indicated that the accuracy is very good and error rate was $\sim 0.01 \%$ (Fig. 1A). The GC content was $\sim 50 \%$ besides the first $1-15 \mathrm{bp}$, which was removed before further analysis (Fig. 1B). The sequencing depth in all samples ranged between 10,000x and 750,000x, and most amplications were $>20,000 x$ (Fig. 1C).

Concordance of tumor DNA and matched plasma cfDNA sample. All detected mutations are listed in Table I. Considering possible systematic error, we defined mutations with $>0.5 \%$ percentages as positive mutations. Comparing each plasma cfDNA with its matched tumor DNA, concordant mutations were identified in all 10 patients.

For all concordant mutations identified in the 10 patients, mutation percentages were higher in plasma cfDNA (average $12.04 \%$ ) than in tumor DNA (average 10.80\%) (Table I). Mutations of 26 alleles located in 7 genes (PTEN, STK11, FGFR1, TP53, NOTCH1, ALK and MET) were identified in both tumor DNA and plasma cfDNA. Mutations in EGFR (24.72\% and $18.84 \%)$, KRAS (5.74\%), and FGFR2 (2.02\%) were only identified in tumor DNA while no mutated genes 
Table I. Mutations detected in tumor DNA and plasma cfDNA of NSCLC patients.

\begin{tabular}{|c|c|c|c|c|c|c|c|}
\hline Patient ID & Position & Gene & Mutation & $\begin{array}{l}\text { Mutation } \\
\text { type }\end{array}$ & $\begin{array}{l}\text { Blood mutation } \\
\text { rate (reads) }\end{array}$ & $\begin{array}{l}\text { cfDNA mutation } \\
\text { rate (reads) }\end{array}$ & $\begin{array}{l}\text { Tumor mutation } \\
\text { rate (reads) }\end{array}$ \\
\hline NSCLC1 & chr10:89624218 & PTEN & p.L171V & SUB & 9.01 (11538) & 0.07 (108648) & $0.02(21955)$ \\
\hline NSCLC1 & chr8:38285913 & FGFR1 & p.D133D & DEL & $5.60(1340)$ & $2.22(13695)$ & 2.59 (9729) \\
\hline NSCLC1 & chr9:139399408 & NOTCH1 & p.L1579L & DEL & $1.81(22782)$ & $0.70(153196)$ & 1.03 (34967) \\
\hline NSCLC2 & chr10:89720705 & PTEN & p.T459A & SUB & 0.13 (3197) & $1.04(220526)$ & $0.14(2876)$ \\
\hline NSCLC2 & chr19:1221293 & STK11 & p.Y272Y & SUB & 0.05 (11956) & $2.54(1434088)$ & $0.38(11433)$ \\
\hline NSCLC2 & chr2:29443617 & ALK & p.A1200A & SUB & 0 (107957) & $0(1566735)$ & 49.29 (69422) \\
\hline NSCLC2 & chr7:116339672 & MET & p.S178S & SUB & $0.09(4232)$ & $0.58(504813)$ & $0.56(3416)$ \\
\hline NSCLC2 & chr7:55259515 & EGFR & p.L858R & SUB & $0.01(43374)$ & $0.01(421187)$ & $24.72(34533)$ \\
\hline NSCLC2 & chr8:38285913 & FGFR1 & p.D133D & DEL & $2.60(12201)$ & 3.46 (170139) & 3.10 (7457) \\
\hline NSCLC2 & chr9:139399408 & NOTCH1 & p.L1579L & DEL & $0.94(33155)$ & $1.11(806291)$ & $1.05(22104)$ \\
\hline NSCLC3 & chr17:7577538 & TP53 & p.R209L & SUB & $0.01(65412)$ & $0.01(668018)$ & $3.61(62283)$ \\
\hline NSCLC3 & chr17:7579476 & TP53 & p.P32S & SUB & 20.06 (325417) & $16.12(112725)$ & 3.75 (266676) \\
\hline NSCLC3 & chr8:38285913 & FGFR1 & p.D133D & DEL & $2.92(20513)$ & $2.22(14258)$ & $2.96(20809)$ \\
\hline NSCLC3 & chr9:139399408 & NOTCH1 & p.L1579L & DEL & $0.94(46337)$ & 0.67 (162970) & $1.05(46751)$ \\
\hline NSCLC4 & chr10:89624218 & PTEN & p.L171V & SUB & $12.84(14043)$ & $51.14(3154)$ & 53.31 (12299) \\
\hline NSCLC4 & chr19:1223125 & STK11 & p.F354L & SUB & 3.02 (48133) & $56.19(46898)$ & $34.46(40610)$ \\
\hline NSCLC4 & chr3:178938796 & PIK3CA & p.V680L & SUB & $0.44(4980)$ & $2.13(47)$ & $0.24(13656)$ \\
\hline NSCLC4 & chr8:38285913 & FGFR1 & p.D133D & DEL & 4.97 (3017) & 3.14 (8777) & $2.72(7789)$ \\
\hline NSCLC4 & chr9:139399408 & NOTCH1 & p.L1579L & DEL & $1.68(33691)$ & $0.67(150)$ & $1.05(17506)$ \\
\hline NSCLC5 & chr17:7577565 & TP53 & p.N200S & SUB & $0.05(27965)$ & $0.10(386509)$ & $15.33(46046)$ \\
\hline NSCLC5 & chr2:29443617 & ALK & p.A1200A & SUB & $47.93(63513)$ & $51.31(335544)$ & $42.54(104652)$ \\
\hline NSCLC5 & chr3:178936091 & PIK3CA & p.E545K & SUB & $0(0)$ & $0.73(33218)$ & $21.64(5084)$ \\
\hline NSCLC5 & chr8:38285913 & FGFR1 & p.D133D & DEL & $3.04(7144)$ & $2.34(36438)$ & $3.35(15840)$ \\
\hline NSCLC6 & chr10:123279651 & FGFR2 & p.G172R & SUB & $0.03(22113)$ & $0.02(146276)$ & 2.02 (30992) \\
\hline NSCLC6 & chr12:25398285 & KRAS & p.G12C & SUB & $0.01(34200)$ & $0.01(144676)$ & $5.74(37623)$ \\
\hline NSCLC6 & chr17:7578406 & TP53 & p.R136H & SUB & $0.07(27351)$ & 0.05 (164157) & $2.64(37926)$ \\
\hline NSCLC6 & chr8:38285913 & FGFR1 & p.D133D & DEL & $2.90(10253)$ & $2.39(30748)$ & $3.01(16766)$ \\
\hline NSCLC7 & chr8:38285913 & FGFR1 & p.D133D & DEL & $2.44(6968)$ & $2.63(53550)$ & $3.03(7382)$ \\
\hline NSCLC7 & chr9:139399408 & NOTCH1 & p.L1579L & DEL & $0.82(33227)$ & $0.82(149833)$ & $1.01(28019)$ \\
\hline NSCLC8 & chr17:7578272 & TP53 & p.H154Y & SUB & $0.04(26414)$ & $0.24(330592)$ & $28.81(17584)$ \\
\hline NSCLC8 & chr7:116339672 & MET & p.S178S & SUB & 49.17 (7269) & $50.86(181222)$ & $43.71(4985)$ \\
\hline NSCLC8 & chr7:116340262 & MET & p.N375S & SUB & $50.06(21006)$ & $50.47(213064)$ & 44.18 (13086) \\
\hline NSCLC8 & chr8:38285913 & FGFR1 & p.D133D & DEL & $2.74(21644)$ & 3.26 (188537) & $2.76(13826)$ \\
\hline NSCLC8 & chr9:139399408 & NOTCH1 & p.L1579L & DEL & $0.96(53785)$ & $1.10(543910)$ & $0.96(42236)$ \\
\hline NSCLC9 & chr10:89624218 & PTEN & p.L171V & SUB & $9.05(4155)$ & $0.01(297185)$ & $0.06(16938)$ \\
\hline NSCLC9 & chr3:178938796 & PIK3CA & p.V680L & SUB & $1.14(9864)$ & $0.23(304805)$ & $0.21(21326)$ \\
\hline NSCLC9 & chr8:38285913 & FGFR1 & p.D133D & DEL & $5.11(2054)$ & $3.32(229921)$ & $2.79(12710)$ \\
\hline NSCLC9 & chr9:139399408 & NOTCH1 & p.L1579L & DEL & 1.65 (23599) & $1.14(439058)$ & $0.84(31467)$ \\
\hline NSCLC10 & chr17:7578534 & TP53 & p.K93N & SUB & $0.05(13254)$ & $0.04(279526)$ & $14.22(8318)$ \\
\hline NSCLC10 & chr7:55259515 & EGFR & p.L858R & SUB & $0.02(46073)$ & 0.17 (368684) & $18.84(36517)$ \\
\hline NSCLC10 & chr8:38285913 & FGFR1 & p.D133D & DEL & $3.02(14564)$ & $3.37(146536)$ & $2.59(9555)$ \\
\hline NSCLC10 & chr9:139399408 & NOTCH1 & p.L1579L & DEL & $1.10(34408)$ & $1.09(532945)$ & $0.77(25075)$ \\
\hline
\end{tabular}

found in plasma cfDNA but not in tumor DNA, indicated that plasma cfDNA could partially reflect the genetic condition of NSCLC tumors.
Differences in mutation pattern among tumor tissue, cfDNA and blood of NSCLC. To reveal the association between mutation pattern of tumor tissue and cfDNA, unclustered heatmap 

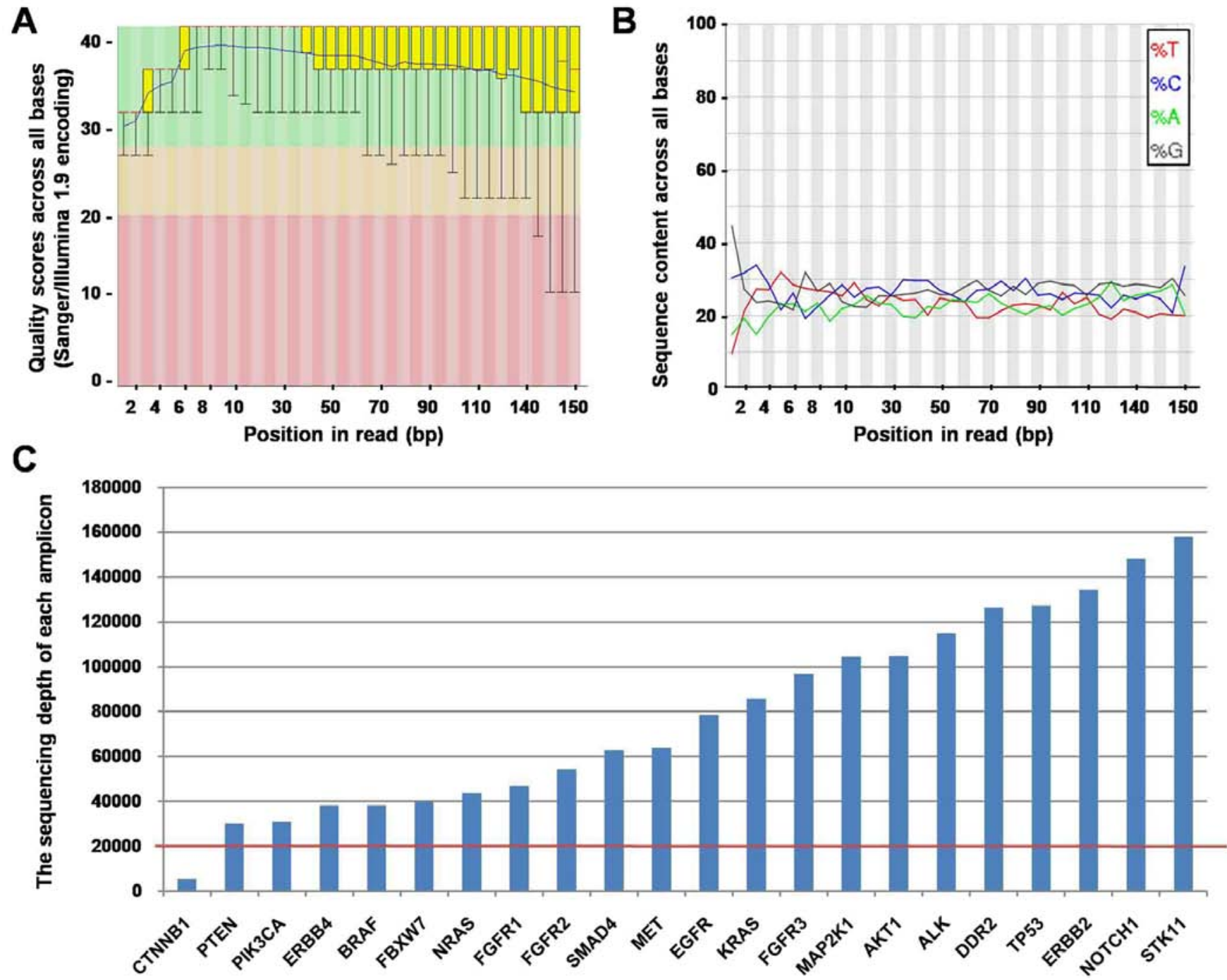

Figure 1. Sequence coverage with the Illumina X10. (A) Quality scores across all bases (every base has a quality score which indicates the accuracy of sequencing). (B) GC content across all bases. (C) The sequencing depth of each amplicon, most amplicons were $>20,000 \mathrm{x}$ (showed by a red line).

diagrams were displayed for mutation patterns of tumor tissue, cfDNA and blood (Fig. 2A). It is clearly showed that cfDNA have a moderate mutation pattern, which is weaker than that of tumor tissue and stronger than that of blood. Mutation patterns of different tissues from the same patients were clustered by hierarchical clustering algorithm (Fig. 2B). For each representative patient, plasma cfDNA was closely clustered with tumor DNA not blood DNA, indicated that plasma cfDNA was associated with tumor genetically.

Mutations in plasma cfDNA sample of healthy people. It is widely known that mutations in NSCLC patients could be caused by senescence and smoking. To further investigate the source and specificity of mutations in NSCLC plasma cfDNA, we recruited 13 elderly people and 7 middle-age smokers and subjected their plasma cfDNA samples to sequencing.

The plasma cfDNA concentration of NSCLC patients $(69.2 \pm 46.9 \mathrm{ng} / \mathrm{ml})$ is significantly higher than that of elderly people $(32.5 \pm 5.2 \mathrm{ng} / \mathrm{ml}, \mathrm{t}=2.96, \mathrm{p}=0.007)$ and middle-aged smokers $(17.9 \pm 9.1 \mathrm{ng} / \mathrm{ml}, \mathrm{t}=2.83, \mathrm{p}=0.013)$. Violin plot of plasma cfDNA concentration is shown in Fig. 3A.
Nearly half of mutations (PTEN_c.511C $>$ G, STK11_ c. $1062 \mathrm{C}>\mathrm{G}, \mathrm{ALK}$ _c. $3600 \mathrm{C}>\mathrm{G}, \mathrm{MET}$ _c. $534 \mathrm{C}>\mathrm{T}$, MET_c.1124A $>$ G, NOTCH1_c.4735G $>$ G) were identified in both NSCLC patients and healthy controls (elderly people and smokers). However, five mutations (PTEN_c.1375A $>$ G, TP53_c.94G>A, STK11_c.816C >T, PIK3CA_c.1633G>A, PIK3CA_c.2038G>C) only identified in NSCLC patients, indicated that cfDNA mutation pattern was largely different between NSCLC patients and non-cancerous people.

\section{Discussion}

The 5-year survival rate following surgical resection for NSCLC at early stage is significantly higher than that at late stage $(2,19)$. Unfortunately, NSCLC is always diagnosed at advanced stages because the symptoms are not apparent initially and the detection is difficult at stage I or II $(20,21)$. Thus, exploring an effective approach to detect early-stage patients can observably increase survival. Even though biopsy has been the golden diagnostic method, in many cases of NSCLC, it is always difficult to obtain tissue samples in early- 
A

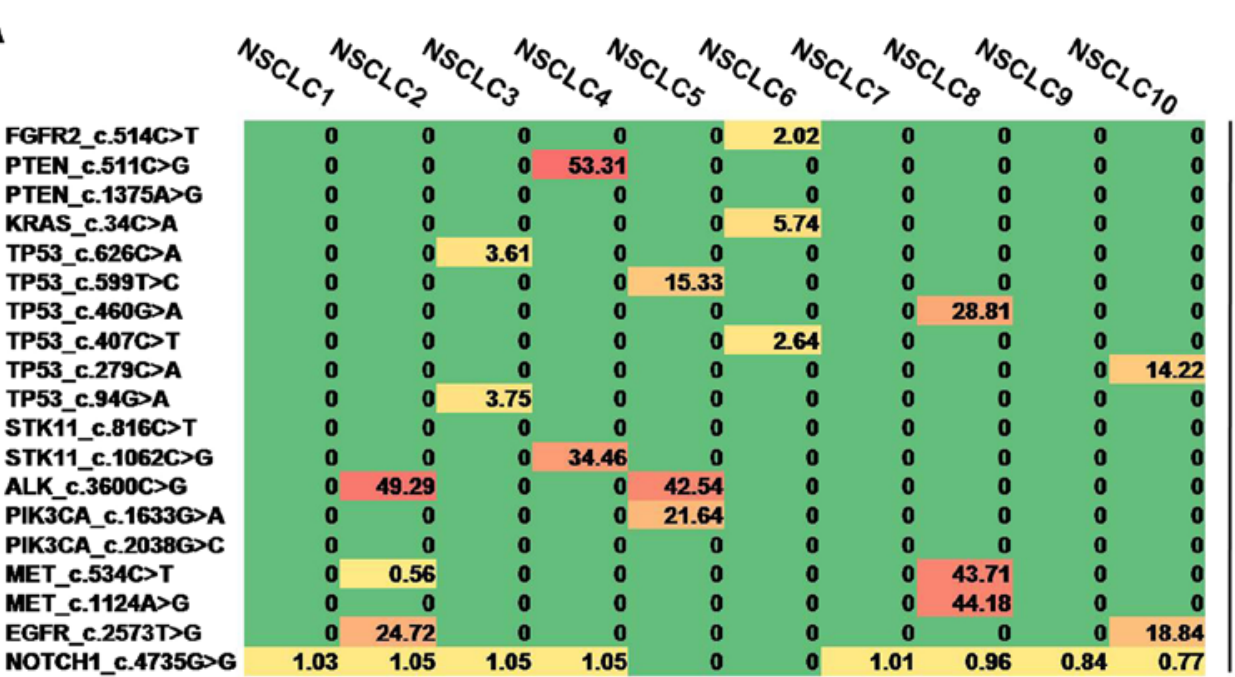

B
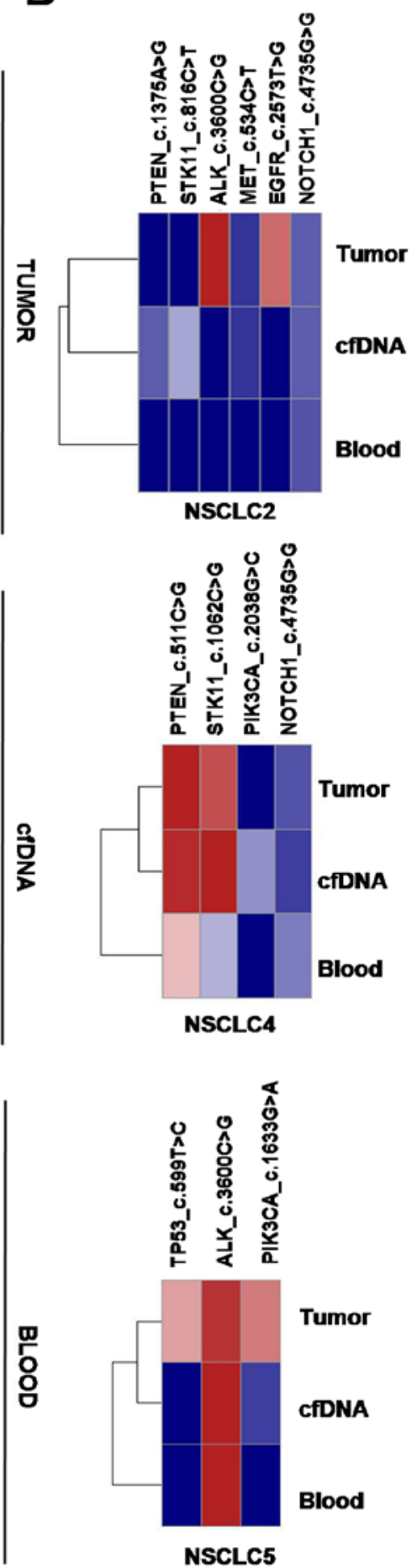

Figure 2. Differences in mutation pattern among tumor tissue, cfDNA and blood of NSCLC patients. (A) Frequencies of all mutated alleles in all samples, high frequency mutations are red and low frequency mutations are green (upper panel, tumor tissue; middle panel, cfDNA; lower panel, blood). (B) Hierarchical clustering by mutation pattern in tumor tissue, cfDNA and blood of single representative NSCLC patient (upper panel, NSCLC patient 2; middle panel, NSCLC patient 4; lower panel, NSCLC patient 5).

stage or even in advanced or metastatic stage (18). Additionally, the procedure of biopsy may increase the risk of cancer 'seeding' to other sites (22). In recent years, 'liquid biopsy' receives more and more attention but most studies involved in mutations of NSCLC are about late stages. Therefore the detection of liquid biopsy biomarkers in early-stage NSCLC patients is necessary and can provide a non-invasive way to gain genotypic information and will have broad application prospects in the large-scale in the future (18).

Several hypotheses have been proposed to explain the mechanism of cellular DNA release into the circulation. $\mathrm{Wu}$ et al proposed that tumor DNA was released into the circulation and was enriched in the plasma and serum and the increased cfDNA in plasma of cancer patients may be due 

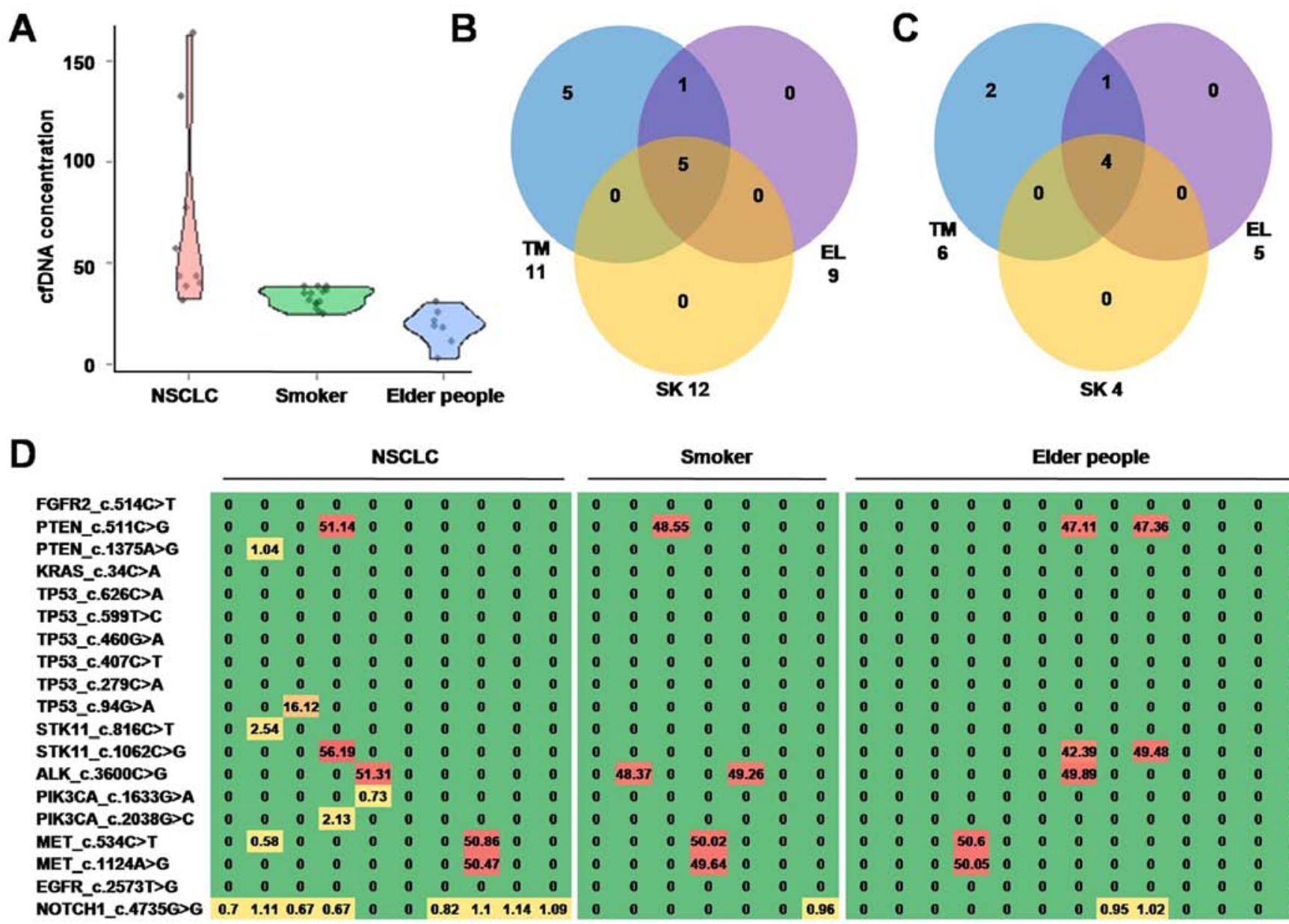

\begin{tabular}{ccccccccccccc}
10 & 110 \\
\hline 0 & 0 & 0 & 0 & 0 & 0 & 0 & 0 & 0 & 0 & 0 & 0 & 0 \\
0 & 0 & 0 & 0 & 0 & 0 & 47.11 & 0 & 47.36 & 0 & 0 & 0 & 0 \\
0 & 0 & 0 & 0 & 0 & 0 & 0 & 0 & 0 & 0 & 0 & 0 & 0 \\
0 & 0 & 0 & 0 & 0 & 0 & 0 & 0 & 0 & 0 & 0 & 0 & 0 \\
0 & 0 & 0 & 0 & 0 & 0 & 0 & 0 & 0 & 0 & 0 & 0 & 0 \\
0 & 0 & 0 & 0 & 0 & 0 & 0 & 0 & 0 & 0 & 0 & 0 & 0 \\
0 & 0 & 0 & 0 & 0 & 0 & 0 & 0 & 0 & 0 & 0 & 0 & 0 \\
0 & 0 & 0 & 0 & 0 & 0 & 0 & 0 & 0 & 0 & 0 & 0 & 0 \\
0 & 0 & 0 & 0 & 0 & 0 & 0 & 0 & 0 & 0 & 0 & 0 & 0 \\
0 & 0 & 0 & 0 & 0 & 0 & 0 & 0 & 0 & 0 & 0 & 0 & 0 \\
0 & 0 & 0 & 0 & 0 & 0 & 0 & 0 & 0 & 0 & 0 & 0 & 0 \\
0 & 0 & 0 & 0 & 0 & 0 & 42.39 & 0 & 49.48 & 0 & 0 & 0 & 0 \\
0 & 0 & 0 & 0 & 0 & 0 & 49.89 & 0 & 0 & 0 & 0 & 0 & 0 \\
0 & 0 & 0 & 0 & 0 & 0 & 0 & 0 & 0 & 0 & 0 & 0 & 0 \\
0 & 0 & 0 & 0 & 0 & 0 & 0 & 0 & 0 & 0 & 0 & 0 & 0 \\
0 & 0 & 0 & 50.6 & 0 & 0 & 0 & 0 & 0 & 0 & 0 & 0 & 0 \\
0 & 0 & 0 & 50.05 & 0 & 0 & 0 & 0 & 0 & 0 & 0 & 0 & 0 \\
0 & 0 & 0 & 0 & 0 & 0 & 0 & 0 & 0 & 0 & 0 & 0 & 0 \\
0 & 0 & 0 & 0 & 0 & 0 & 0 & 0.95 & $\mathbf{1 . 0 2}$ & 0 & 0 & 0 & 0 \\
\hline
\end{tabular}

Figure 3. Differences in cfDNA mutation pattern among NSCLC patients, smokers and elderly people. (A) Plasma cfDNA concentration of NSCLC patients, smokers and elderly people. (B) Venn diagram showed overlaps in mutated alleles among NSCLC patients, smokers and elderly people. (C) Venn diagram showed overlaps in mutated genes among NSCLC patients, smokers and elderly people. (D) Frequencies of all mutated alleles in all cfDNA samples, high frequency mutations are red and low frequency mutations are green (left panel, NSCLC patients; middle panel, smokers; right panel, elderly people).

to the derivation from tumor DNA (23). Lee and colleagues hypothesized that the cfDNA was composed of small fragments of nucleic acid that are not associated with cells or cell fragments and should be from a leakage after tumor necrosis or apoptosis (24).

Jamal-Hanjani et al found there were ubiquitous and heterogeneous single-nucleotide variants (SNVs) between tumor DNA and cfDNA in lung cancer, incidence of which are $68 \%$ and $32 \%$, respectively (25). Couraud et al reported that not all tumor mutations could be detected in cfDNA (50 mutations were identified in tumor DNA, while only 26 were detected in cfDNA) (26). Further detection of mutations in tumor DNA and cfDNA was required to define its potential use in clinical practice. In this study, we recruited 10 NSCLC patients of early stage (includes IA, IB, IIA and IIB); collected matched DNA samples and conducted further research. We found that tumor DNA and its matched plasma cfDNA samples showed high concordance in their mutation patterns.

Notably, some specific mutations were identified in tumor cfDNA, locating in genes of EGFR, KRAS and FGFR2, which is not common in lung cancer tumor samples. These results are also coherent with precious studies $(17,27)$. However, the incidence of mutations in plasma cfDNA was roughly higher than that in tumor DNA in some previous studies $(18,28)$. In this study, plasma cfDNA had a moderate mutation pattern, which was weaker than that of tumor tissue and stronger than that of PBLs. Generally, plasma cfDNA was closely clustered with tumor DNA not blood DNA, indicating that plasma cfDNA was associated with tumor genetically.

Many studies proposed that cfDNA concentration can reflect the tumor burden in the cases of cancer (26). In cancer patients, a relatively high concentration of cfDNA (range $0-1,000 \mathrm{ng} / \mathrm{ml}$, average $180 \mathrm{ng} / \mathrm{ml}$ ) were reported (29) compared to that of health people (range $0-100 \mathrm{ng} / \mathrm{ml}$, average $30 \mathrm{ng} / \mathrm{ml}$ ) (30), which may be predominantly originated from apoptotic cells, inflammation, tissue trauma and so on (31). Some studies even revealed that cfDNA concentration was different between early and late stages (26).

Association between mutations of cfDNA with those of tumor tissue DNA was also observed in prostate cancer and breast cancer $(32,33)$. cfDNA is not only associated with carcinogenesis. Its concentration may also be elevated in inflammatory, infectious and other health-related conditions 
such as senescence and smoking status (34). Thus further studies need to establish a standard to distinguish potential lung cancer patients with healthy subjects.

Our results suggested that the concentrations of cfDNA in NSCLC patients showed significant differences when compared with that of elderly people $(t=2.96, p=0.007)$ and that of middle-aged smokers $(\mathrm{t}=2.83, \mathrm{p}=0.013)$ although the median concentration of cfDNA can vary with senescence and smoking among healthy subjects (35). Therefore our data may suggest that the quantification of cfDNA from plasma may be a useful non-invasive technique for diagnosis and dynamic monitoring of lung cancer at early stage.

In some circumstances cfDNA alterations are detectable ahead of cancer diagnosis, raising the possibility of exploiting them as biomarkers for monitoring cancer occurrence (36). Although detection of mutations in cfDNA is difficult due to the low amount of mutant alleles in a background of wild-type DNA (37), there are still five mutations (PTEN_c.1375A $>$ G, TP53_c.94G >A, STK11_c.816C >T, PIK3CA_c.1633G >A, PIK3CA_c.2038G >C) identified in NSCLC patients specifically, suggesting that cfDNA mutation pattern could distinguish early NSCLC patients with non-cancerous individuals. Stankovic et al reported that coexistence of aberrant p53 and PTEN was the most frequent marker and significantly associated with poor survival of NSCLC patients (38). Stjernström et al also found when analyzing all PI3K pathway related genes together, NSCLC patients would have at least one alteration (39). STK11 mutation was first found in PeutzJeghers syndrome patients, which is significantly associated with KRAS and EGFR (40). Mutation in STK11 was also found associated with prognosis (41).

In conclusion, we find that cfDNA has a similar mutation pattern with its matched tumor tissue DNA. Specific mutations or elevated concentration of plasma cfDNA could be useful non-invasive biomarkers through liquid biopsy in prevention and diagnosis of NSCLC. However, future investigation in utility and perspectives of cfDNA still need to be performed in large prospective cohorts.

\section{Acknowledgements}

This study was supported by grants from the National Natural Scientific Foundation of China (81272682) and the Financial department of Hebei Province (no. [2014]1257).

\section{References}

1. Catarino R, Coelho A and Medeiros R: Circulating DNA and NSCLC: Old findings with new perspectives. J Thorac Dis 4: 442-443, 2012.

2. Torre LA, Bray F, Siegel RL, Ferlay J, Lortet-Tieulent J and Jemal A: Global cancer statistics, 2012. CA Cancer J Clin 65: 87-108, 2015.

3. Islami F, Torre LA and Jemal A: Global trends of lung cancer mortality and smoking prevalence. Transl Lung Cancer Res 4: 327-338, 2015.

4. Torre LA, Siegel RL, Ward EM and Jemal A: International variation in lung cancer mortality rates and trends among women. Cancer Epidemiol Biomarkers Prev 23: 1025-1036, 2014.

5. Chen W, Zheng R, Baade PD, Zhang S, Zeng H, Bray F, Jemal A, Yu XQ and He J: Cancer statistics in China, 2015. CA Cancer J Clin 66: 115-132, 2016.
6. Reade CA and Ganti AK: EGFR targeted therapy in non-small cell lung cancer: Potential role of cetuximab. Biologics 3 : 215-224, 2009.

7. Agarwal M, Brahmanday G, Chmielewski GW, Welsh RJ and Ravikrishnan KP: Age, tumor size, type of surgery, and gender predict survival in early stage (stage I and II) non-small cell lung cancer after surgical resection. Lung Cancer 68: 398-402, 2010.

8. Aberle DR, Adams AM, Berg CD, Black WC, Clapp JD, Fagerstrom RM, Gareen IF, Gatsonis C, Marcus PM and Sicks JD; National Lung Screening Trial Research Team: Reduced lung-cancer mortality with low-dose computed tomographic screening. N Engl J Med 365: 395-409, 2011.

9. Church TR, Black WC, Aberle DR, Berg CD, Clingan KL, Duan F, Fagerstrom RM, Gareen IF, Gierada DS, Jones GC, et al; National Lung Screening Trial Research Team: Results of initial low-dose computed tomographic screening for lung cancer. N Engl J Med 368: 1980-1991, 2013.

10. Lai Y, Shen C, Wang X, Du H, Chen D, Tian L, Zhou X and Che G: Status and perspectives of detection by low-dose computed tomography or computed radiography in surgical patients with lung cancer, based on a five-year study. Thorac Cancer 7: 111-117, 2016.

11. Zhao SJ and Wu N: Early detection of lung cancer: Low-dose computed tomography screening in China. Thorac Cancer 6: 385-389, 2015.

12. Silva M, Galeone C, Sverzellati N,, Marchianò A, Calareso G, Sestini S, La Vecchia C, Sozzi G, Pelosi G and Pastorino U: Screening with low-dose computed tomography does not improve survival of small cell lung cancer. J Thorac 11: 187-193, 2016.

13. Tissot C, Toffart AC, Villar S, Souquet PJ, Merle P, Moro-Sibilot D, Pérol M, Zavadil J, Brambilla C, Olivier M, et al: Circulating free DNA concentration is an independent prognostic biomarker in lung cancer. Eur Respir J 46: 1773-1780, 2015.

14. Moyer VA; U.S. Preventive Services Task Force: Screening for lung cancer: U.S. Preventive Services Task Force recommendation statement. Ann Intern Med 160: 330-338, 2014.

15. Humphrey L, Deffebach M, Pappas M, et al: U.S. Preventive Services Task Force Evidence Syntheses, formerly Systematic Evidence Reviews. In: Screening for Lung Cancer: Systematic Review to Update the U.S. Preventive Services Task Force Recommendation Agency for Healthcare Research and Quality (US), Rock ville, MD, 2013.

16. Sun W, Yuan X, Tian Y, Wu H, Xu H, Hu G and Wu K: Non-invasive approaches to monitor EGFR-TKI treatment in non-small-cell lung cancer. J Hematol Oncol 8: 95, 2015.

17. Nygaard AD, Garm Spindler KL, Pallisgaard N, Andersen RF and Jakobsen A: The prognostic value of KRAS mutated plasma DNA in advanced non-small cell lung cancer. Lung Cancer 79: 312-317, 2013.

18. Guo K, Zhang Z, Han L, Han J, Wang J, Zhou Y, Liu H, Tong L, Li X and Yan X: Detection of epidermal growth factor receptor mutation in plasma as a biomarker in Chinese patients with early-stage non-small cell lung cancer. Onco Targets Ther 8: 3289-3296, 2015.

19. Parkin DM, Bray F, Ferlay $\mathbf{J}$ and Pisani P: Estimating the world cancer burden: Globocan 2000. Int J Cancer 94: 153-156, 2001.

20. Rossi A, Maione P, Colantuoni G, Gaizo FD, Guerriero C, Nicolella D, Ferrara C and Gridelli C: Screening for lung cancer: New horizons? Crit Rev Oncol Hematol 56: 311-320, 2005.

21. Brambilla C, Fievet F, Jeanmart M, de Fraipont F, Lantuejoul S, Frappat V, Ferretti G, Brichon PY and Moro-Sibilot D: Early detection of lung cancer: Role of biomarkers. Eur Respir J (Supplement 39): S36-S44, 2003.

22. Murtaza M, Dawson SJ, Pogrebniak K, Rueda OM, Provenzano E, Grant J, Chin SF, Tsui DW, Marass F, Gale D, et al: Multifocal clonal evolution characterized using circulating tumour DNA in a case of metastatic breast cancer. Nat Commun 6: 8760, 2015.

23. Wu TL, Zhang D, Chia JH, Tsao K, Sun CF and Wu JT: Cell-free DNA: Measurement in various carcinomas and establishment of normal reference range. Clin Chim Acta 321: 77-87, 2002.

24. Lee YJ, Yoon K-A, Han J-Y, Kim HT, Yun T, Lee GK, Kim HY and Lee JS: Circulating cell-free DNA in plasma of never smokers with advanced lung adenocarcinoma receiving gefitinib or standard chemotherapy as first-line therapy. Clin Cancer Res 17: 5179-5187, 2011.

25. Jamal-Hanjani M, Wilson GA, Horswell S, Mitter R, Sakarya O, Constantin T, Salari R, Kirkizlar E, Sigurjonsson S, Pelham R, et al: Detection of ubiquitous and heterogeneous mutations in cell-free DNA from patients with early-stage non-small-cell lung cancer. Ann Oncol 27: 862-867, 2016. 
26. Couraud S, Vaca-Paniagua F, Villar S, Oliver J, Schuster T, Blanché H, Girard N, Trédaniel J, Guilleminault L, Gervais R, et al: Noninvasive diagnosis of actionable mutations by deep sequencing of circulating free DNA in lung cancer from neversmokers: A proof-of-concept study from BioCAST/IFCT-1002. Clin Cancer Res 20: 4613-4624, 2014.

27. Duan H, Lu J, Lu T, Gao J, Zhang J, Xu Y, Wang M, Wu H, Liang $\mathrm{Z}$ and Liu T: Comparison of EGFR mutation status between plasma and tumor tissue in non-small cell lung cancer using the Scorpion ARMS method and the possible prognostic significance of plasma EGFR mutation status. Int J Clin Exp Pathol 8: 13136-13145, 2015.

28. Jung K, Fleischhacker M and Rabien A: Cell-free DNA in the blood as a solid tumor biomarker - a critical appraisal of the literature. Clin Chim Acta 411: 1611-1624, 2010.

29. Shapiro B, Chakrabarty M, Cohn EM and Leon SA: Determination of circulating DNA levels in patients with benign or malignant gastrointestinal disease. Cancer 51: 2116-2120, 1983.

30. Anker P and Stroun M: Circulating DNA in plasma or serum. Medicina (B Aires) 60: 699-702, 2000.

31. Schwarzenbach H, Hoon DS and Pantel K: Cell-free nucleic acids as biomarkers in cancer patients. Nat Rev Cancer 11: 426-437, 2011.

32. Feng J, Gang F, Li X, Jin T, Houbao H, Yu C and Guorong L: Plasma cell-free DNA and its DNA integrity as biomarker to distinguish prostate cancer from benign prostatic hyperplasia in patients with increased serum prostate-specific antigen. Int Urol Nephrol 45: 1023-1028, 2013.

33. Huang ZH, Li LH and Hua D: Quantitative analysis of plasma circulating DNA at diagnosis and during follow-up of breast cancer patients. Cancer Lett 243: 64-70, 2006.

34. Ulivi P and Silvestrini R: Role of quantitative and qualitative characteristics of free circulating DNA in the management of patients with non-small cell lung cancer. Cell Oncol (Dordr) 36 439-448, 2013.
35. Huang R, Wei Y, Hung RJ, Liu G, Su L, Zhang R, Zong X, Zhang ZF, Morgenstern H, Brüske I, et al: Associated links among smoking, chronic obstructive pulmonary disease, and small cell lung cancer: A pooled analysis in the International Lung Cancer Consortium. EBioMedicine 2: 1677-1685, 2015.

36. Gormally E, Caboux E, Vineis P and Hainaut P: Circulating free DNA in plasma or serum as biomarker of carcinogenesis: Practical aspects and biological significance. Mutat Res 635: 105-117, 2007.

37. Sherwood JL, Corcoran C, Brown H, Sharpe AD, Musilova M and Kohlmann A: Optimised pre-analytical methods improve KRAS mutation detection in circulating tumour DNA (ctDNA) from patients with non-small cell lung cancer (NSCLC). PLoS One 11: e0150197, 2016.

38. Stankovic T, Milinkovic V, Bankovic J, Dinic J, Tanic N, Dramicanin $\mathrm{T}$ and Tanic N: Comparative analyses of individual and multiple alterations of p53, PTEN and p16 in non-small cell lung carcinoma, glioma and breast carcinoma samples. Biomed Pharmacother 68: 521-526, 2014.

39. Stjernström A, Karlsson C, Fernandez OJ, Söderkvist P, Karlsson MG and Thunell LK: Alterations of INPP4B, PIK3CA and pAkt of the PI3K pathway are associated with squamous cell carcinoma of the lung. Cancer Med 3: 337-348, 2014.

40. Ylikorkala A, Avizienyte E, Tomlinson IP, Tiainen M, Roth S, Loukola A, Hemminki A, Johansson M, Sistonen P, Markie D, et al: Mutations and impaired function of LKB1 in familial and non-familial Peutz-Jeghers syndrome and a sporadic testicular cancer. Hum Mol Genet 8: 45-51, 1999.

41. Pécuchet N, Laurent-Puig P, Mansuet-Lupo A, Legras A, Alifano M, Pallier K, Didelot A, Gibault L, Danel C, Just PA, et al: Different prognostic impact of STK11 mutations in non-squamous non-small-cell lung cancer. Oncotarget, Nov 25, 2015 (Epub ahead of print). doi: 10.18632/oncotarget.6379. 